\title{
ANÁLISE MODAL DE EDIFICAÇÃO DO SÉCULO XIX
}

\author{
I. SOUSA \\ Estudante de graduação \\ UFC Russas/ LAREB \\ Ceará, Brasil \\ israelnlsousa@gmail.com
}

\author{
F. MINEIRO \\ Estudante de graduação \\ UFC Russas/ LAREB \\ Ceará, Brasil \\ mineironeto1@gmail.com
}

\author{
M. FREIRES \\ Estudante de graduação \\ UFC Russas/ LAREB \\ Ceará, Brasil \\ marcelofreires18@gmail.com
}

\author{
E. MESQUITA \\ Professor, PhD \\ UFC Russas/ LAREB \\ Ceará, Brasil \\ emesquita@ufc.br
}

\author{
P. DAMASCENO \\ Estudante de graduação \\ UFC Russas/ LAREB \\ Ceará, Brasil \\ isaacufcrussas@gmail.com
}

\section{RESUMO}

A conservação de construções históricas tem se tornado objeto de estudo nos últimos anos, principalmente em prol do desenvolvimento de técnicas não destrutivas, que permitem analisar os elementos estruturais causando pouco ou nenhum dano à estrutura. Em função disso, destaca-se a análise estrutural por meio do método dos elementos finitos (MEF), permitindo compreender diferentes tipos de solicitações e seus efeitos sobre o desempenho estrutural dessas construções. Este trabalho trata da análise de uma estrutura de pedra construída no século XIX, na cidade de Quixadá no Ceará. Assim, a modelagem numérica foi realizada com o auxílio do software ANSYS®, versão 17.1, possibilitando uma caracterização dinâmica da construção. Logo, os resultados deste trabalho permitiram obter informações acerca do seu desempenho estrutural, como as frequências naturais, deslocamentos e modos de vibração, contribuindo no estudo da sua estabilidade e em potenciais alternativas de intervenção nessa construção.

Palavras-chave: construções históricas, modelagem numérica, caracterização dinâmica.

\section{ABSTRACT}

Conservation of historical constructions has become the object of study in recent years, mainly due to the development of non-destructive techniques, which allow the analysis of structural elements causing little or no damage to the structure. As a result, the structural analysis by the finite element method (MEF) stands out, allowing to understand different types of requests and their effects on the structural performance of these constructions. This work deals with the analysis of a stone structure built in the 19th century, in the city of Quixadá, in Ceará. Thus, numerical modeling was performed with the aid of ANSYS ${ }^{\circledR}$ software, version 17.1, enabling a dynamic characterization of the construction. Consequently, the results of this work provided information on its structural performance, such as natural frequencies, displacements and vibration modes, contributing to the study of its stability and potential intervention alternatives in this construction.

Keywords: historical constructions, numerical modeling, dynamic characterization.

\section{INTRODUÇÃO}

As construções históricas caracterizam-se como parte do patrimônio histórico material de uma sociedade, desempenhando assim, relevante papel no âmbito artístico, cultural, religioso, documental ou estético de um povo (BRANDÃO et al., 2018). Dessa forma, essas construções permitem compreender características acerca de um passado comum, bem como entender os diferentes mecanismos responsáveis pelo desenvolvimento dessa comunidade. Assim como a maioria das edificações, essas construções também estão submetidas a ações externas em função da própria natureza, antrópicas e relativas à efeitos dinâmicos, como do vento ou de sismos.

A avaliação dos danos em construções, define a necessidade ou não de intervenção por meio de manutenção ou reabilitação, em função disso, surgem trabalhos relacionados à caracterização de materiais, como apresentado por 
(SILVEIRA; VARUM; COSTA, 2013), que consistiu na caracterização de tijolos de adobe produzidos na cidade de Aveiro, em Portugal.

Portanto, a dificuldade em realizar a caracterização de danos em construções históricas, remete aos problemas encontrados nas etapas de avaliação, no que diz respeito principalmente à obtenção de informações acerca das características dos materiais utilizados e dos métodos construtivos implementados na execução da obra. Em função disso, com o passar dos anos foram desenvolvidas e implementadas normas técnicas e códigos construtivos objetivando a padronização dos métodos construtivos. À vista disso, foi então desenvolvido o boletim técnico n ${ }^{\circ} 11$ (MESQUITA et al., 2015), com o objetivo de auxiliar eventuais avaliações de danos em construções históricas, permitindo uma correta abordagem frente a análise de danos nessas edificações.

Essa dificuldade de análise das características construtivas e de materiais permitiu, portanto, o desenvolvimento de técnicas não destrutivas, na caracterização dos materiais componentes das construções históricas, como o ensaio ultrassônico, responsável pela obtenção de propriedades como a velocidade de pulso ultrassônico, meio pelo qual torna possível a identificação de características como a resistência à compressão e o módulo de elasticidade do concreto. No entanto, mesmo com a implementação dessa técnica, ainda existem dificuldades na caracterização de alguns materiais, como as alvenarias de argila e de pedra (NOOR-E-KHUDA; ALBERMANI, 2019),(KOROTH; FAZIO; FELDMAN, 1998), (ALIABDO; ELMOATY, 2012), bem como na caracterização de painéis de alvenaria (MESQUITA et al., 2018), contribuindo numa melhor análise acerca das características desses materiais. No entanto, com a ausência do equipamento para análise, muitas vezes recorre-se ao uso do método dos elementos finitos, buscando atribuir uma análise estática ou dinâmica na caracterização global de uma estrutura.

As ações dinâmicas estão relacionadas essencialmente às solicitações que variam ao longo do tempo e existência de movimentos oscilatórios, definidos por vibrações. Dessa forma, evidencia-se que a caracterização dessas ações se relaciona à obtenção de frequências naturais e modos de vibrações. A determinação dessas propriedades torna-se, portanto, objeto de estudo recorrente, evidenciado principalmente por ensaios realizados in situ, como a utilização de sistemas de monitoramento óptico em edificações (MESQUITA et al., 2016), assim como a abordagem relativa à modelagem numérica na obtenção das propriedades dinâmicas dos materiais. A importância da caracterização da influência de ações dinâmicas nas construções define efeitos nas características de serviço dos elementos estruturais em análise, tornando-se um relevante meio de avaliação utilizado nas construções históricas, também graças a possibilidade de realização do procedimento de caracterização dessas propriedades sem danificar a estrutura.

A preservação do patrimônio histórico, em especial as construções históricas, tem se tornado objeto de estudo nos últimos anos, necessitando de profunda investigação em função do seu desempenho estrutural. Isso possibilita o desenvolvimento de técnicas e medidas de investigação da estabilidade estrutural das construções, como os ensaios ultrassônicos, sistemas de monitoramento óptico e estudo das modelagens numéricas.

Deste modo, o desenvolvimento de estudos acerca das características de serviço do edificado histórico tem ganhado relevância. Devido a isso, foi realizada uma análise dinâmica acerca de um galpão construído em alvenaria de argila e pedra, situado na cidade de Quixadá, no Ceará, no século XIX, com o objetivo de contribuir no desenvolvimento técnico-científico e possibilitando ampliação de conhecimento acerca da preservação do patrimônio histórico, em especial as construções de influência luso-brasileira.

\section{ESTUDO DE CASO}

\subsection{Açude do Cedro}

A necessidade por abastecimento de água, e desenvolvimento da agricultura, foram determinantes na construção do Açude do Cedro situado em Quixadá, no estado do Ceará, dessa forma, surgiu como uma tentativa de solucionar a problemática da seca enfrentada pela região (MONTEIRO, 2012). A construção desse açude datou do ano de 1884 a 1906 e possibilitou ainda a construção de outras edificações para o auxílio do desenvolvimento da obra, dentre essas construções destaca-se o galpão de armazenamento de materiais, evidenciado na figura 1.

O presente trabalho buscou, dessa forma, realizar a caracterização dinâmica do galpão de armazenamento de materiais, buscando analisar as características dessa construção histórica, bem como avaliar a estabilidade estrutural frente as ações dinâmicas. 


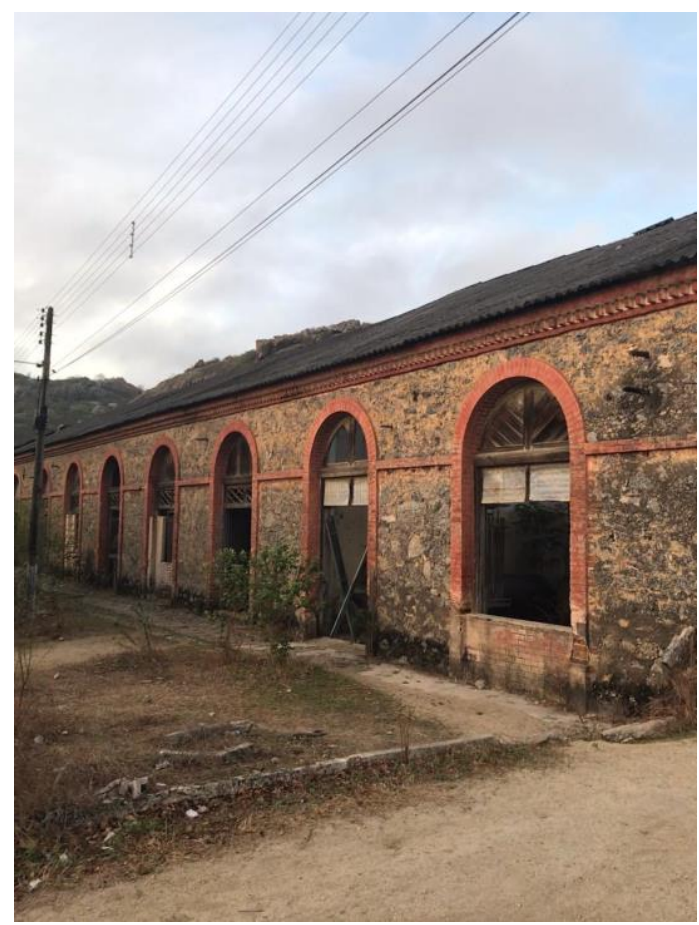

Figura 1. Galpão de armazenamento de materiais do Açude do Cedro.
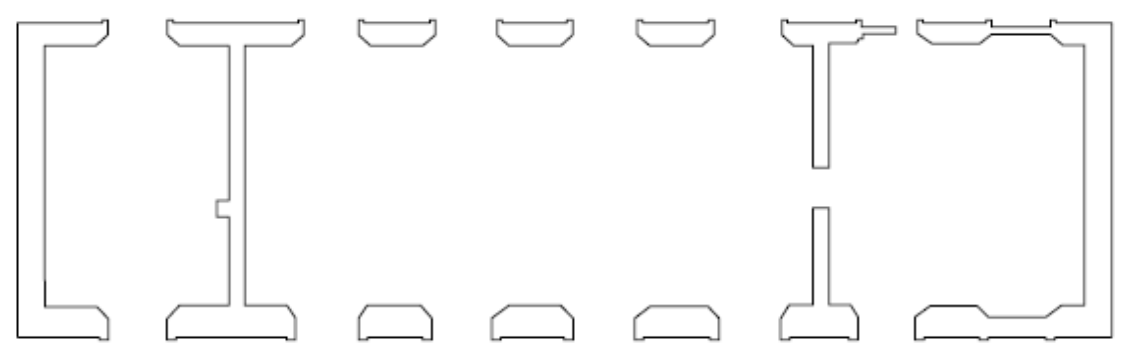

Figura 2. Planta baixa representativa do galpão de armazenamento de materiais.

O galpão de armazenamento de materiais se configura como uma construção mista de alvenaria e pedra. Dessa forma, foi construído com a intenção de auxiliar nas obras da construção do Açude do Cedro. Essencialmente, essa construção foi tombada, junto a todo o complexo de construções, em 1977, pelo Instituto do Patrimônio Histórico e Artístico Nacional (IPHAN).

\section{CARACTERIZAÇÃo DA ESTRUTURA}

\subsection{Método dos elementos finitos}

O método dos elementos finitos definiu a possibilidade de uma nova abordagem nos problemas de engenharia. Dessa forma, ele consiste na discretização de estruturas, permitindo sua redução em pequenos elementos interligados por meio de pontos nodais, que em conjunto formam as malhas de elementos finitos. Os comportamentos individuais de cada um desses elementos são então superpostos aos demais, permitindo obter características acerca do comportamento global da estrutura em análise. No galpão em estudo, ocorre a predominância do elemento Solid187 dentre os elementos utilizados na análise. Ele se caracteriza essencialmente por ser um elemento de 10 nós, com três graus de liberdade em cada um dos nós, totalizado assim, 67.330 elementos e 120.095 nós.

As estruturas podem então ser caracterizada quanto ao tipo de solicitação as quais são submetidas, definidas em estáticas e dinâmicas. As estáticas definem as solicitações que variam ao longo de um intervalo de tempo alto, ou não variam com o tempo, ao passo que as análises dinâmicas buscam compreender os efeitos das solicitações ao longo de 
um intervalo de tempo, uma vez que, nesse tipo de análise, as solicitações variam com o tempo, tornando necessário o estudo do comportamento do material, no momento em que a solicitação está atuante.

\subsection{Análise modal}

$\mathrm{Na}$ análise modal, busca-se definir a estrutura em função da sua frequência natural e dos seus diferentes modos de vibrações (BRANDÃO et al., 2016). Dessa forma, torna-se possível determinar, por meio dessa análise, um modelo matemático que justifique os efeitos das solicitações variantes com o tempo, por meio do método dos elementos finitos.

Portanto, a frequência natural é compreendida como uma taxa de oscilação pela qual a estrutura passa a ser submetida a partir do momento em que é cessada a solicitação. Já os modos de vibrações dizem respeitos às diferentes possibilidades de movimentos oscilatórios que a estrutura pode apresentar, relacionadas diretamente com a frequência natural.

\subsection{Carregamentos e propriedades mecânicas das alvenarias da análise em estudo}

Recorreu-se à literatura na obtenção das solicitações atuantes na construção, uma vez que, a ausência dessas informações fez-se evidente ao longo do desenvolvimento do trabalho. A obtenção dessas características permitiu de forma adequada, encontrar as propriedades mecânicas relativas aos materiais utilizados na construção.

O módulo de elasticidade (E) foi obtido para a alvenaria de pedra e cerâmica, por meio do regulamento italiano "Norme Tecniche per le costruzioni”, de 2018 (MIT, 2018). O peso específico (w) e a resistência à compressão (fc) também foram determinadas pelo regulamento italiano de 2018. A resistência à tração (ft), foi obtida com base no trabalho de (BRANDÃO, 2017), bem como o coeficiente de Poisson, obtidos com base nos trabalhos de (BRANCO, M, E, 2007), (FRAZÃO, 2013) e (BRANDÃO, 2017). A tabela 1 determina as propriedades dos materiais encontradas.

Tabela 1. Magnitudes das propriedades mecânicas obtidas na literatura.

\begin{tabular}{ccc}
\hline Propriedades & Alvenaria de pedra & Alvenaria cerâmica \\
\hline \hline $\mathrm{E}(\mathrm{GPa})$ & 4,75 & 1,7 \\
$\mathrm{w}\left(\mathrm{KN} / \mathrm{m}^{3}\right)$ & 22 & 18 \\
$\mathrm{fc}(\mathrm{MPa})$ & 4,75 & 3,2 \\
$\mathrm{ft}(\mathrm{MPa})$ & 0,24 & 0,16 \\
$\mathrm{v}$ & 0,2 & 0,2 \\
\hline
\end{tabular}

Além dos valores apresentados, para a cobertura, através do trabalho de (NEVES, S, M, L, 2008), foi obtido valor igual a a $1,3 \mathrm{kN} / \mathrm{m}^{2}$, considerando todos os elementos constituintes (telha, ripas, caibros e treliças). Segundo a NBR 6120 (ABNT, 1980) faz-se necessário a utilização de uma carga acidental equivalente a $0,5 \mathrm{kN} / \mathrm{m}^{2}$. Superpondo os carregamentos, obtemos $1,8 \mathrm{kN} / \mathrm{m}^{2}$ para a cobertura, no entanto, segundo (BRANDÃO, 2017), faz-se necessário majorar a carga em 50\%, resultando em um carregamento final relativo à cobertura de $2,7 \mathrm{kN} / \mathrm{m}^{2}$.

Tabela 2. Representação dos carregamentos referentes à cobertura.

\begin{tabular}{cc}
\hline Carregamentos & Representação \\
\hline \hline $1,3 \mathrm{kN} / \mathrm{m}^{2}$ & Telhas, ripas, caibros e treliças \\
$0,5 \mathrm{kN} / \mathrm{m}^{2}$ & Sobrecarga \\
$2,7 \mathrm{kN} / \mathrm{m}^{2}$ & Majoração \\
\hline
\end{tabular}

\section{RESULTADOS E DISCUSSÕES}

Com a realização da análise modal, foi possível identificar os 20 primeiros modos de vibrações do galpão de armazenamento, bem como suas respectivas frequências naturais e períodos, juntamente às suas porcentagens de massas modais efetivas em cada uma das direções analisadas.

Analisando as direções X, Y e Z, a soma das porcentagens das massas modais efetivas em cada um dos eixos correspondeu a respectivamente, $64,70 \%, 45,22 \%$ e 0,015\%. Dessa forma, a direção X é a responsável pelas maiores 
deformações modais, uma vez que existe a participação da massa modal pode ser tomada como uma importante variável no comportamento global de uma estrutura. Fica evidente também que a maioria dos valores de massas modais efetivas são maiores na direção X, bem como o maior valor dessa propriedade, localizado no modo 5 (Tabela 3), com 15,803\%.

Na direção Y também foi percebida a influência nos modos de vibrações, uma vez que apresentou massa modal efetiva total equivalente a 45,22\%, com o modo 1 sendo o responsável pela maior influência na composição desse valor.

Na direção Z, ficou evidente a ausência de influência nos modos de vibrações, uma vez que os valores respectivos são muito baixos, estando próximos a zero.

Tabela 3. Valores das propriedades encontradas na análise modal

\begin{tabular}{|c|c|c|c|c|c|}
\hline \multirow{2}{*}{ Modo } & \multirow{2}{*}{$\begin{array}{c}\text { Frequência } \\
\mathbf{f}(\mathbf{H z})\end{array}$} & \multirow{2}{*}{$\frac{\text { Período }}{\text { T (s) }}$} & \multicolumn{3}{|c|}{ Massa modal efetiva } \\
\hline & & & $\mathrm{Ux}(\%)$ & $\mathbf{U y}(\%)$ & $\mathrm{Uz}(\%)$ \\
\hline 1 & 5,607 & 0,178 & 0,062 & 14,099 & 0,000 \\
\hline 2 & 6,830 & 0,146 & 5,420 & 4,273 & 0,000 \\
\hline 3 & 7,281 & 0,137 & 1,977 & 8,160 & 0,004 \\
\hline 4 & 8,004 & 0,124 & 0,829 & 0,332 & 0,000 \\
\hline 5 & 8,624 & 0,115 & 15,803 & 0,061 & 0,000 \\
\hline 6 & 9,177 & 0,108 & 3,399 & 0,131 & 0,000 \\
\hline 7 & 9,922 & 0,100 & 1,132 & 3,886 & 0,000 \\
\hline 8 & 10,215 & 0,097 & 0,109 & 0,041 & 0,000 \\
\hline 9 & 10,438 & 0,095 & 0,204 & 3,478 & 0,001 \\
\hline 10 & 12,540 & 0,079 & 0,093 & 1,926 & 0,000 \\
\hline 11 & 12,745 & 0,078 & 1,066 & 0,106 & 0,000 \\
\hline 12 & 13,223 & 0,075 & 0,062 & 5,042 & 0,005 \\
\hline 13 & 14,410 & 0,069 & 1,689 & 0,002 & 0,000 \\
\hline 14 & 16,118 & 0,062 & 0,161 & 0,039 & 0,000 \\
\hline 15 & 17,131 & 0,058 & 2,687 & 0,083 & 0,000 \\
\hline 16 & 17,736 & 0,056 & 2,415 & 0,644 & 0,000 \\
\hline 17 & 19,067 & 0,052 & 6,126 & 0,041 & 0,000 \\
\hline 18 & 19,756 & 0,050 & 9,705 & 0,056 & 0,000 \\
\hline 19 & 20,330 & 0,049 & 4,927 & 2,798 & 0,000 \\
\hline 20 & 20,607 & 0,048 & 6,826 & 0,016 & 0,000 \\
\hline
\end{tabular}

Com a Tabela 3, torna-se possível compreender que à medida que ocorre o aumento nos modos de vibrações, é observado também um aumento na frequência natural, bem como uma redução gradual do período. Evidencia-se também que a frequência natural referente ao modo 1, equivalente a 5,607 Hz, é compreendida como a mais importante dentre as demais, uma vez que é a menor em magnitude, indicando uma maior possibilidade de ser atingida por efeitos externos, como sismos ou ventos, e quando essa frequência natural é atingida, pode ocorrer o fenômeno da ressonância, podendo ocasionar o colapso da estrutura.

Na figura 3, percebe-se os seis primeiros modos de vibrações, através dela torna-se possível identificar que nos modos 1, 2, 3 e 4 ocorre a predominância de excitação dos deslocamentos nas alvenarias laterais. Também se evidencia que os modos 2, 3, 4 e 6 ocorre excitação do deslocamento da parede interna. Já quanto à fachada frontal, o deslocamento é excitado apenas nos modos 5 e 6 , como mostra figura 3 .

As deformações modais máximas encontradas na parede de alvenaria lateral, foram nos modos 1 e 4 , evidenciadas na Tabela 4, com valores iguais à $0,23 \mathrm{~mm}$ e $0,22 \mathrm{~mm}$, respectivamente. Na parede interna, a deformação máxima foi encontrada no modo 2, e equivalente a $0,25 \mathrm{~mm}$. Já na fachada frontal, evidenciou-se a presença de deformação máxima no modo 5, equivalente em magnitude a $0,225 \mathrm{~mm}$. 


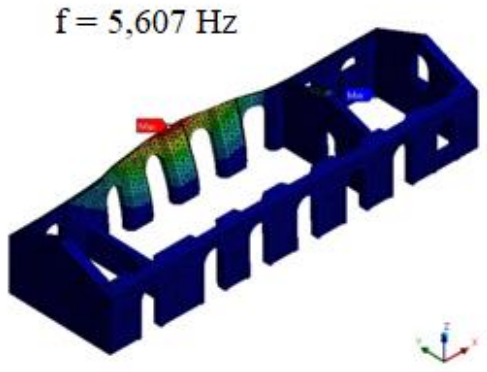

(a)

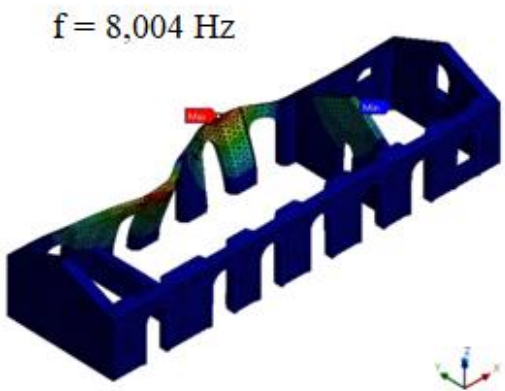

(d)

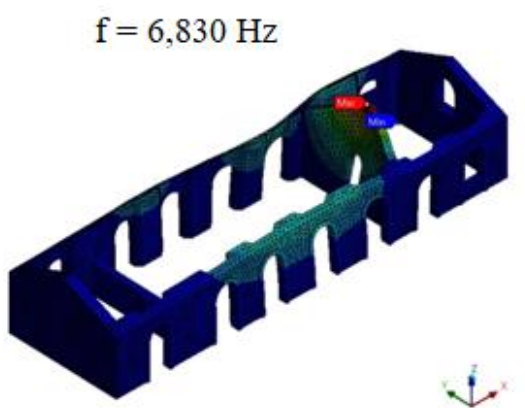

(b)

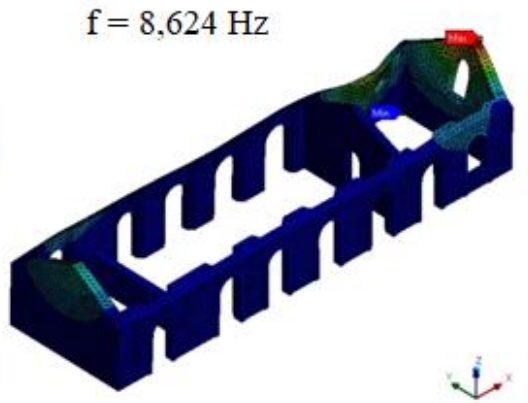

(e)

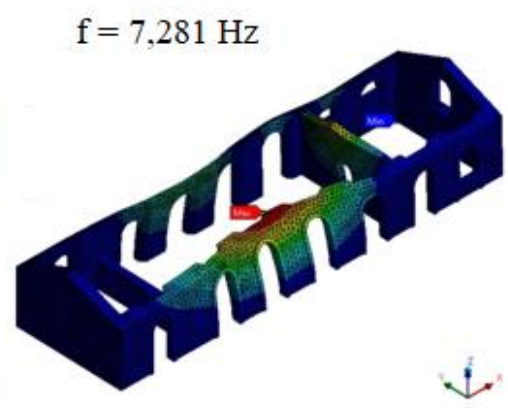

(c)

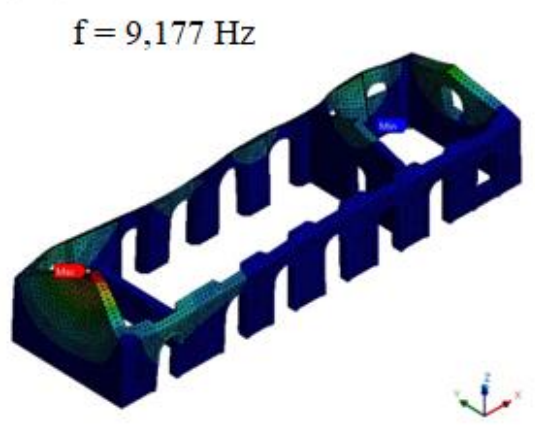

(f)

Figura 3. Modos de vibração; (a) Modo 1; (b) Modo 2; (c) Modo 3; (d) Modo 4; (e) Modo 5; (f) Modo 6.

Dessa forma, as paredes laterais apresentam uma maior vulnerabilidade em relação aos demais elementos, no entanto ressalta-se também os efeitos dos deslocamentos nas fachadas frontal e posterior, identificando possíveis danos a esses elementos, caso a frequência natural desses modos venha a ser atingida.

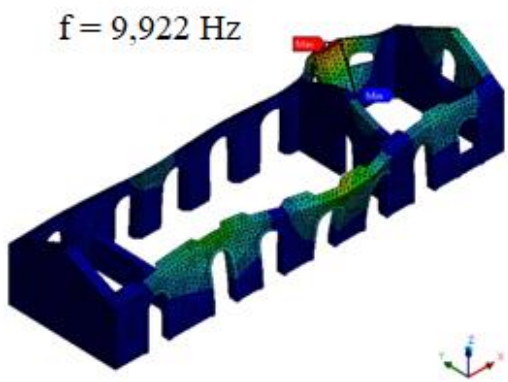

(a)

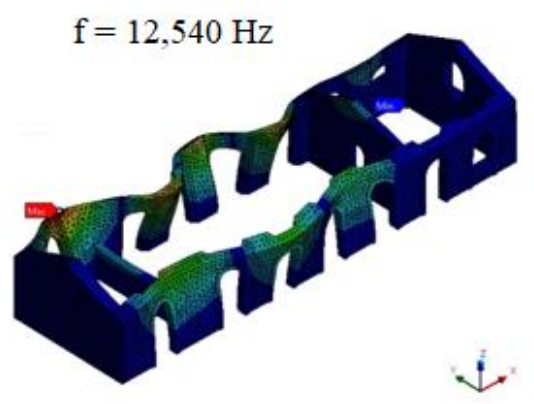

(d)

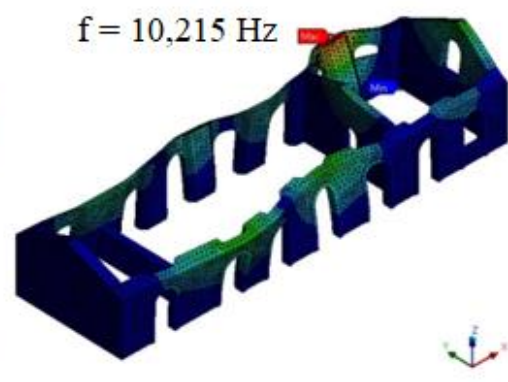

(b)

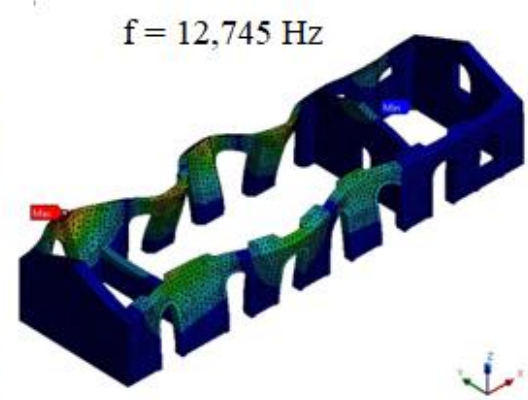

(e)

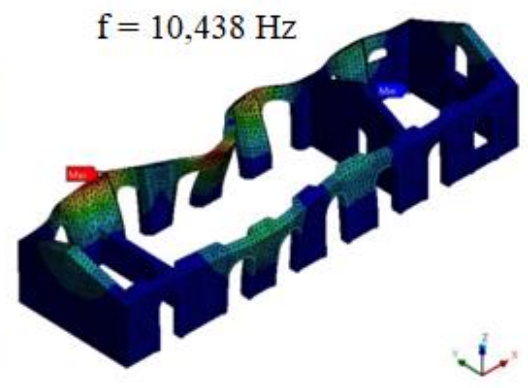

(c)

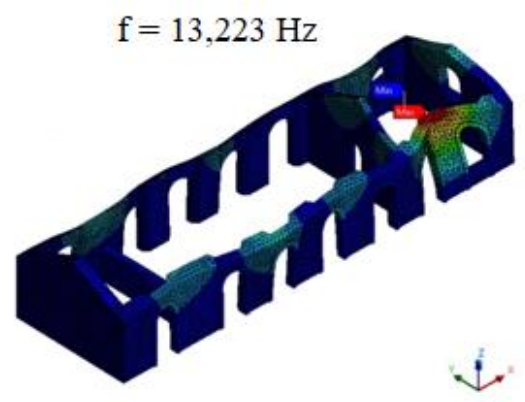

(f)

Figura 4. Modos de vibração; (a) Modo 7; (b) Modo 8; (c) Modo 9; (d) Modo 10; (e) Modo 11; (f) Modo 12. 
Tabela 4. Deformações máximas e mínimas nos modos de vibrações.

\begin{tabular}{ccc}
\hline Modo & Deformação máxima $(\mathbf{m m})$ & Deformação mínima $(\mathbf{m m})$ \\
\hline \hline 1 & 0,23008 & 0,00000 \\
2 & 0,25351 & 0,00000 \\
3 & 0,16762 & 0,00000 \\
4 & 0,22035 & 0,00000 \\
5 & 0,22511 & 0,00000 \\
6 & 0,19041 & 0,00000 \\
7 & 0,21514 & 0,00000 \\
8 & 0,20212 & 0,00000 \\
9 & 0,17874 & 0,00000 \\
10 & 0,17543 & 0,00000 \\
11 & 0,13726 & 0,00000 \\
12 & 0,23413 & 0,00000 \\
13 & 0,50525 & 0,00000 \\
14 & 0,15066 & 0,00000 \\
15 & 0,16469 & 0,00000 \\
16 & 0,22397 & 0,00000 \\
17 & 0,32234 & 0,00000 \\
18 & 0,18729 & 0,00000 \\
19 & 0,14048 & 0,00000 \\
20 & 0,11480 & 0,00000 \\
\hline
\end{tabular}

A figura 4 mostra os modos 7 ao 12, permitindo atribuir maiores movimentações principalmente nas paredes laterais. Dessa forma, nos modos 7, 8, 9, 10 e 11, evidenciou-se maiores movimentações na parede lateral esquerda, já que o modo 12 foi o único a atribuir maior excitação na deformação da parede lateral direita.

Percebe-se também na Tabela 4, que as maiores movimentações dos primeiros 12 modos, foram encontradas nos modos 7 e 12 , com respectivamente $0,21 \mathrm{~mm}$ e $0,23 \mathrm{~mm}$, no entanto, enquanto no modo 7 a deformação ocorre na parede lateral esquerda, no modo 12, a deformação ocorre na parede lateral direita.

\section{CONCLUSÕES}

A inegável participação do Açude do Cedro na história do estado do Ceará traz à tona a necessidade por estuda-lo e preserva-lo, dessa forma, assegurando sua importância como patrimônio histórico. Logo, por meio desse trabalho buscou-se analisar a estabilidade dinâmica do galpão de armazenamento de materiais, através do método dos elementos finitos. Para tal, foi realizada uma visita técnica à obra, com o intuito de executar a caracterização geométrica do local, permitindo a construção dos modelos tridimensionais usados na obtenção dos resultados.

Através da análise foi possível identificar uma maior massa modal efetiva nas direções X e Y, equivalentes a 64,70\% e $45,22 \%$, justificando um maior deslocamento de massa nessas direções. Na direção Z, o valor do deslocamento foi próximo de 0 , uma vez que o próprio travamento de engastamento da estrutura possibilita essa condição.

Foi possível verificar também que a maior deformação ocorre em função do modo 2, com $0,25 \mathrm{~mm}$, na parede interna da construção. Verificou-se ainda que todos os 12 modos de vibração influenciariam deslocamentos nas paredes laterais.

Dessa forma, o presente trabalho busca contribuir no desenvolvimento da utilização de técnicas não destrutivas na caracterização de materiais, permitindo conhecer o comportamento global da estrutura sob o efeito de diferentes solicitações, auxiliando então, nas possíveis intervenções passíveis de ocorrer nas construções analisadas e em especial, no galpão de armazenamento de materiais do Açude do Cedro. 


\section{REFERÊNCIAS}

ABNT. NBR 6120: Cargas para o cáluclo de estruturas de edificações, 1980.

ALIABDO, A. A. E.; ELMOATY, A. E. M. A. Reliability of using nondestructive tests to estimate compressive strength of building stones and bricks. Alexandria Engineering Journal, v. 51, n. 3, p. 193-203, 2012.

BRANCO, M, E, M. Reforço Sísmico de Edifícios de Alvenaria - Aplicação a edifícios "Gaioleiros". [s.l.] Instituto Superior Técnico, Lisboa, 2007.

BRANDÃO, F. et al. Caracterização dinâmica de um edifício histórico do século XIX construído em al. Congresso Brasileiro de Patologia das Construções. Anais...2016

BRANDÃo, F. Aálise do Comportamento Sísmico de uma Construção Histórico do Patrimônio Sobralense. [s.1.] Universidade Estadual Vale do Acaraú - UVA, 2017.

BRANDÃO, F. et al. Dynamic characterization of a heritage construction from 19th century. Ibracon structures and materials journal, v. 11, p. 52-75, 2018.

FRAZÃO, M. T. DE B. R. F. Modelação de um edifício “Gaioleiro"para Avaliação e Reforço Sísmico. [s.l.] Instituto Superior Técnico de Lisboa, 2013.

KOROTH, B. S. R.; FAZIO, P.; FELDMAN, D. Evaluation of Clay Brick Durability Using Ultrasonic Pulse Velocity. v. 4, n. 2, p. 142-147, 1998.

MESQUITA, E. et al. Tehnical Report N 11 - Characterization, evaluation and structural recovery of historic buildings. [s.l: s.n.].

MESQUITA, E. et al. Global overview on advances in structural health monitoring platforms. Journal of Civil Structural Health Monitoring, v. 6, n. 3, p. 461-475, 2016.

MESQUITA, E. et al. Heterogeneity detection of Portuguese-Brazilian masonries through ultrasonic velocities measurements. Journal of Civil Structural Health Monitoring, v. 8, n. 5, p. 847-856, 2018. I Health Monitoring, v. 8, n. 5, p. 847-856, 2018.

MINISTERO DELLE INFRAESTRUTTURE E DEI TRANSPORTI. Norme Tecniche per le Costruzioni, 2018. MONTEIRO, R. F. Um Monumento ao Sertão : ciência , política e trabalho na construção do Açude Cedro (18841906). [s.l: s.n.].

NEVES, S, M, L, C. Análise Sísmica de um Edifício da Baixa Pombalina. [s.l.] Instituto Superior Técnico de Lisboa, 2008.

NOOR-E-KHUDA, S.; ALBERMANI, F. Mechanical properties of clay masonry units: Destructive and ultrasonic testing. Construction and Building Materials, v. 219, p. 111-120, 20 set. 2019.

SILVEIRA, D.; VARUM, H.; COSTA, A. Influence of the testing procedures in the mechanical characterization of adobe bricks. Construction and Building Materials, v. 40, p. 719-728, 2013. 